\title{
COMParitive Study OF The Organizational Structure OF EngineEring StUdent TeAMS AND TEAM EFFECTIVENESS
}

\author{
Radhika R. Kartha, Dr Michael W. Fowler, and Dr Roydon A. Fraser \\ University of Waterloo \\ mfowler@uwaterloo.ca,rafraser@uwaterloo.ca
}

\begin{abstract}
Design-and-build competitions are integral to effective higher engineering education. Yet, there is not much research investigating if the organizational structures of engineering student teams and team effectiveness follow any trends.

This paper delves into the possibility of this correlation by measuring parameters that contribute to effective teams. This research provides data that is used to judge best practices for engineering student teams. The findings from this paper can then be used as a basis for action when the students find a need for organization development in the future. Additionally, this analysis provides insight into teamwork in engineering. This could benefit $4^{\text {th }}$ year design (a.k.a capstone) projects as well as innovative companies with similar settings.

The core contributors to a team's effectiveness are leadership, direction, planning, knowledge transfer, and meetings for engineering student teams. Although parameters like communication and team culture are important, student teams generally have no problems in these areas. By comparing three organizational structures, it is concluded that in general engineering student teams are best when they follow a holocratic or flatter organizational structure as opposed to a strictly flat organizational structure.
\end{abstract}

Keywords: Organizational structures, Student teams, Teamwork, Team effectiveness, Holocratic, Flat

\section{INTRODUCTION}

Engineering Student Teams in universities encourage teamwork and bring together driven students to solve engineering challenges that are posed in the real world. Some of these engineering student teams are formed because of design-and-build competitions such as Midnight Sun Solar Rayce Car Team (that takes part in American Solar Challenge and World Solar Challenge) [1] and some others because of a non-competition goal such as Engineers for Hope (that aims to better the living conditions in third-world countries) [2]. In an institution like the University of Waterloo (UW), there are over twenty engineering student teams that are trying to create innovative solutions to problems posed. The organizational structure of these engineering student teams is organic. This makes it quite interesting since the organizational structure depends on a variety of factors that are mostly driven by the demand of the goal(s) of the competition, the skill sets of the existing core members of the team as well as lessons learned by the team in the past. None of the teams interviewed had organizational structures that were mandated by organizers, although some of them were provided with a recommended structure. Once these teams found the organizational structure they wanted to follow, team effectiveness parameters were measured, for example leadership, planning, and formal communication through meetings.

The motivation to do a thematic analysis of the organizational structures and team effectiveness stemmed from the fact that all the authors of the paper have been personally involved with engineering student teams at varying capacities. One author, Radhika Kartha works as the Project Manager for the University of Waterloo Alternative Fuels Team (UWAFT) [3]. The other authors, Dr Roydon Fraser and Dr Michael Fowler have been UWAFT's faculty advisors for the past 21 years. Also, the faculty authors have mentored several hundred $4^{\text {th }}$ year engineering design capstone projects throughout their teaching careers. The characterization of teamwork in an engineering setting helps give insight to one of the accreditation criteria stated by the Canadian Engineering Accreditation Board (CEAB). Specifically, CEAB recognizes teamwork, "preferably in a multidisciplinary setting" as an important criterion for engineering [4].

Previous papers have explained that design-based learning provides students with better retention of engineering concepts amongst other benefits. Several papers over the years have done studies in order to establish the importance of experiential learning over just classroom-based studies for higher engineering education [5]. Separately, there are many papers and articles that investigate teamwork parameters and laud the importance 
of teamwork in mainly workplace settings [6]. These works were used to identify important metrics to measure team effectiveness.

This paper is novel in its approach to find the relationship between organizational structure to team effectiveness in an engineering student team setting. The aim is to find strategies, practices, and guidelines for engineering student teams by increasing knowledge of the environment that teams thrive in. This is no simple feat since there are many subjective parameters, many of which are discussed in the subsequent sections of the paper. The definitions of various parameters are also discussed for clarity.

A thematic analysis of the collected data is used to draw conclusions and observations concerning teamwork within an engineering setting, and to investigate if there is a correlation between team structure and team effectiveness. This information can then be extended to a start-up or any company with a fast turnover rate and which is innovation and results driven, much like the engineering student teams at UW. Similarly, discussions from this paper can have implications on $4^{\text {th }}$ year design capstone projects.

\section{TYPES OF ORGANIZATIONAL STRUCTURES}

There is a variety of literature available that provides different team taxonomies and types. However, many of these structures are rigid and applicable only to corporations. Organizational structures for student teams are more fluidic and power is not always with the formal leader, but is generally shared. It should also be noted that students are not paid to be on teams and in many cases gain no incentives such as a university credit. This is extra-curricular work that is quite demanding but is chosen by those students who are passionate about the work they do on the team and can manage it with their regular school work load. Hence, a classification that best suits student teams was chosen and only those applied to classify the engineering student teams are presented below [7].

\subsection{Flatter Organization}

This kind of organization is flatter than the traditional hierarchical organization. In hierarchy, communication flows only from the top to the bottom since all the power and information lies atop. In the case of flatter organizations, although there is a management that gets to make decisions for the team, student engagement is better than in a hierarchy. The opinions of those on the lower tier are valued. Signs of a flatter organization include:
- Strong means of communication (usually digital) that helps for the team to collaborate

- Senior team members (based on time spent on team, not academic year in university) make junior members want to work on the team by sharing a form of comradery

- Leaders push power of decision making to other members on the team and not use them just as task following members

- Tiers or levels with distinguishing titles provided

- Characteristic of medium or larger student teams

\subsection{Flat Organization}

This kind of an organization is much like a selfmanaged team. As the name indicates, there are no titles provided to distinguish team members. However, this does not mean that the team does not fall into a natural hierarchy depending on seniority. Accountability is not officially given to one person but is just understood according to the project undertaken. Indicators for this kind of a student team are:

- No formal titles are provided to team members other than those mandated by competition or university such as safety captain, team lead etc.

- Characteristic of smaller teams

\subsection{Holocratic Organization}

This kind of an organization is when decision making is truly distributed since it depends less on the person and more on the role embodied by the team member. Indicators for holocracy include:

- People fill several roles and these roles get updated regularly even if this means that there is just a slight variation

- Information is openly accessible and important decisions are made during meetings.

- A form of hierarchy exists such that the responsible role makes the decision.

- Characteristic of small or medium-sized student teams

\section{TEAM EFFECTIVENESS}

Team effectiveness not only takes the results produced by the team into account but also the overall interaction of the team. A team's output or performance can rely on a number of factors outside of the team's ability and hence is not a good measure of the team's work or teamwork. A 
holistic approach that includes parameters contributing to team performance as well as teamwork was used to understand team effectiveness in this study. The model described by Salas et al and several team questionnaires as well as factors described in previous papers have heavily influenced the team effectiveness survey developed for this study [6][8][9].

\subsection{Terminology for members}

Definitions of the vocabulary used in the survey have been provided to minimize confusion in interpretation.

Core member: Integral experienced members of the team without whom team functioning becomes hard. They hold titles such as leads, managers etc. within the engineering student team.

Junior member: Team members who contribute to the progress of the team's projects and take lead in specific tasks. They are usually upcoming members and/or coops with the team who have a taste of how the team works and are still training with the team at some capacity.

Volunteer: Team members who have just completed the initial steps, such as safety training, to join the team. However, they still require a lot more direction than a junior member. Although they may help with projects, they are held less accountable than junior members for the project progress.

\subsection{Team Effectiveness Parameters}

Direction: This criterion refers to the team's understanding of its main goal(s). Options on the survey check if there is an understanding of how individual goals work together and align to this main goal.

Leadership: This criterion on the survey understands the attitude of the team leadership better. This is a core parameter for team effectiveness and is weighted appropriately for analysis.

Planning: This criterion measures the schedule planning done by the team and how far it was followed. Many student teams during the interview phase of the study expressed difficulty in anticipating the changes in schedule for reallocation of the resources since work done by the members also depended on the school work load which was quite variable for the team members. Hence, questions regarding reallocation of resources was avoided because most of the teams followed an "all-hands-ondeck" approach when necessary instead of planning for it beforehand. Succession planning for new team leadership was identified as an important factor within the planning parameter for team effectiveness.

Meetings and Reporting: This criterion checks for the effectiveness of the technical communication and the cadence for team meetings. Most of the student teams make use of digital means for communication. However, without specific face-to-face meetings, most of the teams agree that information may not get shared with everyone at the right time.

Knowledge Transfer: This criterion measures the processes and system in place for the student team to successfully pass on lessons learned or information obtained about certain parts or systems. Despite this criterion not showing up in many questionnaires or papers referred, this parameter is of paramount importance on an engineering student team. Without this, the wheel can get reinvented a few times within the same year. This is particularly true in UW with the existence of the alternating work terms and study terms that provides more room to lose information as students transition from one term to the next. This may not contribute as a main parameter for teamwork but definitely affects the team performance due to low productivity.

Multidisciplinary: As the name suggests, this criterion checks for how multidisciplinary the team is. Although not viewed as a core parameter for team effectiveness in generalized papers on teamwork, it is seen as an important factor in industrial settings. The ability to communicate with a diverse set of professionals is an aspect that is encouraged by the CEAB.

Team culture: This parameter taps on the interpersonal relationship between the different team members. This is not a core criterion and is not weighted heavily for analysis. The options or factors under the parameter investigates the coordinating mechanisms of the team.

Team morale': This was yet another parameter like team culture that is not a major contributor but is a coordinating mechanism that explores the team's trust within each other amongst other factors.

Team performance: This parameter inspects the team's outputs (deadlines met, team milestones achieved timely) and ability to seek and provide feedback.

\section{DATA COLLECTION METHODOLOGY}

It was considered that this study be done across multiple universities taking part in the same competition so that the complexity of the goal would remain the same. However, arranging for multiple engineering student teams in the same competition and finding teams that used different organizational structures proved to be difficult. Many of the teams initially approached seemed to use similar organizational structures. Hence, in order to be able to observe different organizational structures, the option of different engineering student teams within the same university was selected.

This meant that all the teams had the same pool of resources to share and also shared similar difficulties. For instance, the UW engineering program has a mandatory coop program which means that students go through 
alternating study terms and work terms. These work terms can be in different parts of the world leaving the engineering student team with two groups of students working towards the same goal for the year. This additional realm of difficulty uniformly affects the teams in this particular study. Since it was hard to quantify the difficulty posed by goals of a team taking part in a specific competition compared to one that was competing in a different challenge, it was assumed that the relative difficulty of the competition was proportional to the amount of human resources on the team. It was also assumed that any other differences between the engineering student teams were offset by a higher percentage of highly skilled personnel on the team in the form of upper years and graduate students. This would make the comparison of team effectiveness between the different student teams reasonably fair.

This study with volunteer based engineering student teams, namely those seen in Table 1 was done in two parts. This was so that the study attained both depth and breadth using quantitative as well as qualitative information.

Table 1: List of the 15 UW Engineering Student Teams that participated in this study.

\begin{tabular}{|l|l|}
\hline UW BAJA SAE & $\begin{array}{l}\text { Eco-Marathon/ } \\
\text { Supermileage }\end{array}$ \\
\hline $\begin{array}{l}\text { Midnight Sun Solar Rayce } \\
\text { Team }\end{array}$ & Engineers without borders \\
\hline UW Formula Motorsports & $\begin{array}{l}\text { International Genetically } \\
\text { Engineered Machine }\end{array}$ \\
\hline $\begin{array}{l}\text { University of Waterloo } \\
\text { Autonomous Sailboat Team }\end{array}$ & UW Robotics \\
\hline $\begin{array}{l}\text { UW Submarine Racing } \\
\text { Team }\end{array}$ & UW Snowmobile Team \\
\hline UWaterloo IISE of Waterloo \\
\hline Waterloo Rocketry Team & $\begin{array}{l}\text { University of Formula Electric } \\
\text { Alternative Fuels Team }\end{array}$ \\
\hline Waterloop & \\
\hline
\end{tabular}

\subsection{Qualitative data collection}

The first part involved talking to the lead(s) of the various teams that volunteered. This interview, with many open-ended questions, helped identify the team's organizational structure as accurately as possible. Other information obtained verbally included, but was not limited to, the number of students on the team (breakdown of lower and upper year, and graduate students), competition details (if applicable), team goals, a description of the team's work culture along with the budget the team worked with (breakdown and source), the team's recruitment activities, and the number of $4^{\text {th }}$ year design capstone projects being done with the team. These sessions took about half an hour and helped establish a personal relationship with the team leads. While concluding the interviews, team leads were given the opportunity to voice their thoughts. Some of them suggested team effectiveness parameters that they thought were important based on their experience while others suggested how recruiting would benefit if students were given credit for their student team work, similar to that given for the $4^{\text {th }}$ year design capstone projects. Minutes from each of these meetings were carefully assimilated for later reference.

\subsection{Quantitative data collection}

The second part of the study involved team effectiveness self-evaluation surveys. These were administered electronically using Google Forms. The team leads leveraged their position within the team to encourage as many team members as possible to take part in this survey.

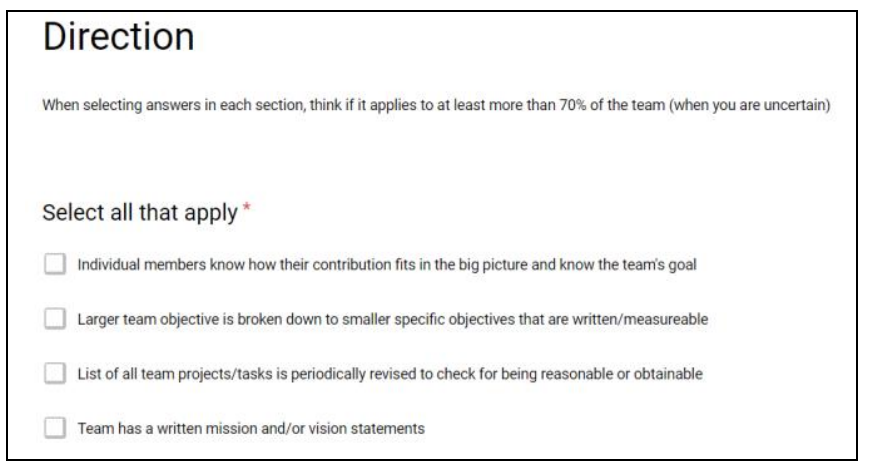

Fig. 1. Sample self-evaluation survey question with four factors

As shown in Fig. 1 above, a selection of factors that helped evaluate a single team effectiveness parameter (Direction here) were offered. All factors that were applicable to each team were selected by each team member.

The benefits of using the survey include the ability to use a standardized data collection method that has a wide reach. It is less time consuming than the interviews which required scheduling at a time both the team lead as well as the authors were available. Since a number of team members from the same team would complete the survey, personal bias was reduced though not eliminated. The surveys allowed for the collection of data from a larger data enabling each parameter to be measured more effectively. However, since this kind of data collection is less accountable than interviews, it is not possible to be absolutely sure how truthfully the students answered the surveys. Measures taken used checkbox answers while a 
comment section was also provided for those interested in elaborating.

\subsection{Analysis of obtained data}

Depending on the characteristics exhibited by the student team, it was grouped under one of the three organizational structures identified in Section 2. It is important to note that no student team was purely flat, flatter than hierarchy or holocratic. Even though the decision relied on data obtained in both phases of the study, it relied more on the first phase as well as first-hand observation of the target team. This helped to assess for which organizational structure the student team would predominantly show characteristics of. Assessment of certain teams can be seen in Table 2 .

Table 2: Organizational Structure of certain teams.

\begin{tabular}{|l|l|}
\hline UWAFT & $\begin{array}{l}\text { Predominantly holocratic; } \\
\text { mildly flatter }\end{array}$ \\
\hline Midnight Sun Solar Rayce & Predominantly flatter \\
\hline Waterloo Rocketry & Predominantly flat \\
\hline $\begin{array}{l}\text { Eco-Marathon/ } \\
\text { Supermileage }\end{array}$ & $\begin{array}{l}\text { Predominantly } \\
\text { migrating to flatter }\end{array}$ \\
\hline $\begin{array}{l}\text { International Genetically } \\
\text { Engineered Machine }\end{array}$ & Predominantly flatter \\
\hline $\begin{array}{l}\text { UW Autonomous Sailboat } \\
\text { Team }\end{array}$ & $\begin{array}{l}\text { Predominantly holocratic; } \\
\text { mildly flat }\end{array}$ \\
\hline
\end{tabular}

Once the organizational structure for all the student teams was established, the results of the survey were anonymized and scrutinized categorically as flatter, flat and holocratic. This pooled the evaluation and mitigated biases characteristic to a single team.

For each organizational structure, if more than $70 \%$ of the pool deemed true for a factor, a collective High was assigned for that factor. Similarly, if $40 \%-70 \%$ was the consensus, the factor would get marked as Medium and if it was less than $40 \%$, a Low was marked on the factor. Once an iteration for each of the organizational structures was completed, the results were further analyzed to find patterns and correlations.

\section{RESULTS AND DISCUSSION}

From the data collected certain trends were observed. The following characteristics are uniform across all engineering student teams irrespective of organizational structure. All teams within UW provide a supportive environment that is encouraging and respectful so that students can voice their opinions. When faced with obstacles, most members try to stay optimistic and avoid blaming each other as much as possible. Project management in terms of schedule management and cost management is not given as much importance as the technical work. This lack of planning and monitoring often hinders team performance. Other than the deliverables or the competition itself, teams do not internally assess their performance and hence have no measure as to whether they are doing better or worse than in previous terms and years (short term or long term). This is attributed to lack of resources and time. The smaller the team, the more the reported data agrees with each other. Most engineering student teams recognize the importance of working in a multidisciplinary setting for their future work. However, this is not a primary contributor or measure of teamwork according to the data collected.

After eliminating the team effectiveness parameters that scored equally high or low on the surveys and interviews immaterial of organizational structure, the parameters that would be used for comparison were established. The five parameters that are weighted to find team effectiveness are leadership, direction, planning, knowledge transfer, and meetings and reporting. Each factor was assigned a weighting as shown in Table 3. As can be seen in Fig. 1., for the one team effectiveness parameter of direction, there are 4 factors that are assessed. Similarly, between all the five team effectiveness parameters, there were 22 factors that are being compared. For a cumulative result, both holocratic organizations as well a flatter organizations scored the same, 19.3 out of 22 . However, flat organizations scored a significantly lesser score of 15 out of 22 .

Table 3: Weighting of factors.

\begin{tabular}{|c|c|}
\hline High & $3 / 3$ \\
\hline Medium & $2 / 3$ \\
\hline Low & $1 / 3$ \\
\hline
\end{tabular}

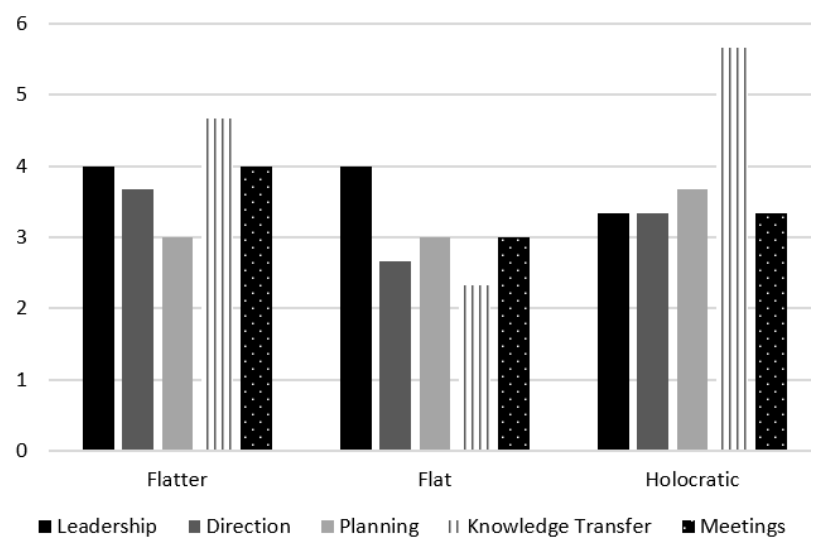

Fig. 2. Chart with results of team effectiveness parameters for different organizational structures. 
Figure 2 shows the differences in parameters between the different organizational structures. Although, holocratic and flatter organizations have a similar overall result, in terms of knowledge transfer and planning, holocratic does better than flatter and flatter does better in terms of leadership, direction and effective meetings. A possible explanation for why knowledge transfer is better in holocracy over a relatively flatter organization is that team members get to do what they choose to do. This would enable them to document and further information on things they are interested to learn. As for having more effective meetings and general team direction when compared to holocratic, flatter organizations are still closer to a hierarchy where these parameters are structured and more rigid.

A major limitation to the results found here is that data was collected from only 48 students. However, it is hoped that the data is sufficient for initial guidance on generalizations and for considering future work. The duration of this study limited the number of participants. Repeating this study over several years is planned. This would help to draw more meaningful conclusions. Also, team performance in terms of awards won can be gauged with records of organizational structure followed at that point.

\section{CONCLUSIONS}

The scores in the results are reflective of how, in an engineering student team setting, some form of organizational structure makes the team more effective. This could be attributed to the fact that in a setting where tasks are performed out of interest, when nobody is explicitly held accountable for the completion of a project or task, the team's productivity can be affected. There may be many other contributing factors as to why a flat structure is not the best for an engineering student team which were not within the scope of this paper. Many teams that were flat in the beginning of the study are trying to migrate to a more hierarchal structured form either because they have more student members on their team now or they have identified some overall defects to the existing team's organizational structure. This practical step validates the empirical data that was collected that separately recommends and concludes that engineering student teams move away from flat structure and follow a flatter or holocratic organization.

Although there is no one best organizational structure that can be recommended to student teams, constantly measuring for team effectiveness can prove useful to gauge if the organizational structure works for the team. Maintaining this adaptability to restructure student teams depending on the transition of team members and other variables is key in being a high performance team.

\section{Acknowledgements}

Sincere gratitude is extended to the EcoCAR 3 Competition for encouraging this research. Also, Ramin Shaikhi is acknowledged as helping significantly with brainstorm, and Maryam Al-Ajeel is to be thanked for helping prepare the survey. The Sedra Student Design Centre and its management is also acknowledged for maintaining records to make it easy to find current team leads of the engineering student teams.

\section{References}

[1] Available as of May 9, 2017 from http://www.uwmidsun.com/

[2] Available as of May 9, 2017 from http://www.engineersforhope.com/

[3] Available as of May 9, 2017 from http://ecocar3.org/waterloo/team-members/

[4] Canadian Engineering Accreditation Board, 2016 Accreditation Criteria and Procedures. 2016 Engineers Canada, 14 pp. \{ISSN 1708-8054\} Available as of May 8, 2017 from https://engineerscanada.ca/sites/default/files/accreditationcriteria-procedures-2016-final.pdf

[5] Sumit Kundu and Michael W. Fowler, "Use of Engineering Design Competitions for Undergraduate and Capstone Projects," Chemical Engineering Education, vol. 43, no. 2, pp. 131-136, 2009.

[6] Eduardo Salas, Dana E. Sims, and C. Shawn Burke, "Is there a "Big Five" in teamwork?" Small Group Research, vol. 36, no.5, pp. 555-599, 2005.

[7] Jacob Morgan, The future of work: Attract new talent, build better leaders, and create a competitive organization. John Wiley \& Sons, 2014. \{ISBN: 978-1-118-87724-1\}

[8] Katia Sycara and Gita Sukthankar, "Literature review of teamwork models," Robotics Institute, Carnegie Mellon University. Pp. 1-18, 2006.

Available as of May 8, 2017 from https://www.researchgate.net/profile/Katia_Sycara2/publicat ion/246704657_Literature_Review_of_Teamwork_Models/1 inks/53d7c9740cf2631430bfc377.pdf

[9] Jon Maxwell, Team Effectiveness Questionnaire 2.0. First Steps Training and Development. Available as of May 8, 2017 from http://www.firststepstraining.com/images/pdf/teq_sample.pd f 